\title{
Plantas Medicinais nos Quintais Urbanos do Município de Orleans, Santa Catarina, Sul do Brasil
}

\author{
Medicinal Plants in Urban Home Gardens in Orleans City, Southern Brazil
}

\author{
Juliana Medeiros Borghezan*a; Angela Erna Rossato ${ }^{a}$; Vanilde Citadini-Zanette ${ }^{\mathrm{a}}$ \\ ${ }^{a}$ Universidade do Extremo Sul Catarinense, Programa de Pós-Graduação Stricto Sensu em Ciências Ambientais. SC, Brasil. \\ *E-mail: borghezanj@outlook.com
}

\begin{abstract}
Resumo
Estudos etnobotânicos possibilitam compreender a forma como as pessoas se relacionam com as plantas, baseado nos saberes populares repassados entre as gerações. Este estudo se caracterizou em um levantamento etnobotânico das plantas medicinais cultivadas nos quintais urbanos dos agentes da Pastoral da Saúde, município de Orleans, Santa Catarina, Brasil. Utilizou-se entrevista semiestruturada, com aplicação de um formulário, com turnê guiada e registro fotográfico, para validação das informações. Foram entrevistados dezenove informantes e registradas 85 espécies pertencentes a 72 gêneros e 39 famílias botânicas, sendo as mais representativas Lamiaceae (13 espécies) e Asteraceae (10 espécies). A folha foi o farmacógeno mais utilizado. As formas terapêuticas de uso mais citadas foram infusão (50,6\%) e decocto (49,4\%). $\mathrm{O}$ estado e forma de uso do farmacógeno utilizados foram fresco e interno, respectivamente. As indicações terapêuticas que prevaleceram foram para enfermidades relacionadas ao trato alimentar e metabolismo, sangue e órgãos hematopoiéticos e sistema respiratório. A origem das plantas, em sua maioria, era exótica. No entanto, algumas espécies nativas estão sendo domesticadas o que poderá incluir plantas em cultivo de pequena escala em futuro próximo. O tempo de uso das plantas medicinais relatado foi mais de 50 anos, sendo a transmissão de forma transgeracional, o que atende os critérios da ANVISA quanto ao registro, com base na tradicionalidade de uso superior a 30 anos. Este estudo reflete o contexto cultural e ambiental enraizado de uma comunidade local, revelando a importância dos quintais como forma social e que pode contribuir para a manutenção dos espaços urbanos, por meio do registro do conhecimento popular.
\end{abstract}

Palavras-chave: Etnobotânica. Pastoral da Saúde. Conhecimento Popular e Científico.

\begin{abstract}
Ethnobotanical studies make it possible to understand the way people relate to plants based on popular knowledge passed from generation to generation. The objective of this study was to perform an ethnobotanical survey of medicinal plants cultivated in the urban backyards of "Pastoral da Saúde" (Health Pastoral) agents, in Orleans municipality, Santa Catarina state, Brazil. A semi-structured interview was used, with application of a questionnaire, complemented with guided tour and photographic record for information validation. Nineteen informants were interviewed. The ethnobotanical survey recorded 85 species belonging to 72 genera and 39 botanical families. The most representative families were Lamiaceae 13 species) and Asteraceae (10 species). The most used pharmacogen was leaf. The most commonly mentioned pharmaceutical forms of use were infusion (50,6\%) and decocted (49,4\%). The state and form of use of the pharmacogen used were fresh and internal, respectively. The prevailing therapeutic indications were diseases related to the nutritional and metabolic problems, blood and hematopoietic organs and respiratory system. The plants' origin was mostly exotic. However, some native species have already been domesticated which may include plants in small-scale cultivation in the near future. The reported time of use was over 50 years, with transmission being transgenerational, which meets ANVISA's criteria for registration, based on traditional use higher to 30 years. This study reflects the rooted cultural and environmental context of a local community, revealing the importance of backyards as a social form and that can contribute to the urban spaces maintenance, through the popular knowledge registration.
\end{abstract}

Keywords: Urban Ethnobotany. Health Pastoral. Folk and Scientific Knowledgment.

\section{Introdução}

Os quintais constituem espaços culturais e sociais, de fácil acesso para os moradores cultivarem uma diversidade de espécies, com amplas finalidades, contemplando um verdadeiro banco de recursos genéticos, de grande relevância para a humanidade (SIVIERO et al., 2012). Desta forma, são considerados importantes meios para a conservação in situ da agrobiodiversidade (ÁVILA et al., 2017). Entretanto, a forma e função dos quintais vêm sendo alteradas e adaptadas às novas exigências socioeconômicas da sociedade moderna, resultantes do processo de urbanização (PERNA; LAMANO-FERREIRA, 2014). Por este motivo, evidencia-se a importância dos estudos etnobotânicos, a fim de conhecer a diversidade de espécies atualmente cultivadas no ambiente urbano e seus usos populares, com base no contexto social (PRADEICZUK et al. (2017) e de autocuidado em saúde. Estes autores relatam ser ainda uma lacuna atual do conhecimento nas pesquisas da etnobotânica brasileira, principalmente no Sul do Brasil. Conforme a análise bibliométrica (1988-2013) realizada por Ritter et al. (2015), os quintais começaram a ser estudados há pouco tempo pelos pesquisadores etnobotânicos 
brasileiros.

Em seu estudo, Duarte et al. (2020) demonstram a importância que os saberes e práticas populares, no uso de plantas medicinais, exercem como recurso primário para o tratamento de várias enfermidades por populações urbanas. Nesse sentido, visando manutenção dos conhecimentos empíricos e a redução da pressão sobre os ecossistemas naturais, Caballero-Serrano et al. (2019) incentivam os formuladores de políticas públicas para que considerem as hortas caseiras como importantes reservatórios de diversidade biológica, cultural e de bem-estar local.

Os agentes pastorais são conhecidos por possuírem vasto conhecimento popular sobre os recursos naturais, disseminado por meio de suas práticas e utilizado para o tratamento de enfermidades, ao longo da história (ROSSATO, 2018). Esses agentes, por meio dos seus quintais, cultivam as matériasprimas ativas vegetais utilizados na preparação de remédios naturais, na troca de mudas e de informações sobre os benefícios das plantas. Conforme Cerqueira et al. (2020), esse conhecimento é transmitido oralmente de geração em geração, por isso emerge a necessidade dos estudos etnobotânicos como instrumento para documentar estes saberes, que podem se perder em função da modernização.

Nesse viés, apoiando-se na premissa de valorizar o saber popular sobre as plantas medicinais e os benefícios ambientais, sociais e econômicos provenientes dos quintais, o presente estudo, de caráter pioneiro, buscou sistematizar as informações de um grupo social engajado na multiplicação e transmissão do conhecimento, em um município escasso de estudos relacionados à temática do trabalho e associados ao público-alvo.

À luz destas indagativas, o objetivo do estudo foi realizar levantamento etnobotânico das plantas medicinais cultivadas nos quintais urbanos dos agentes da Pastoral da Saúde do município de Orleans, Santa Catarina, Sul do Brasil, a fim de registrar as espécies cultivadas, bem como suas finalidades terapêuticas e a origem do conhecimento.

\section{Material e Métodos}

O presente estudo foi realizado no município de Orleans (Figura 1), Santa Catarina, com agentes da Pastoral da Saúde, Regional Sul 4 da Diocese de Tubarão, inserida na Comarca de Braço do Norte (DIOCESE DE TUBARÃO, 2019).

Figura 1 - Localização da área de estudo, com destaque para os bairros estudados do município de Orleans, Santa Catarina, sul do Brasil
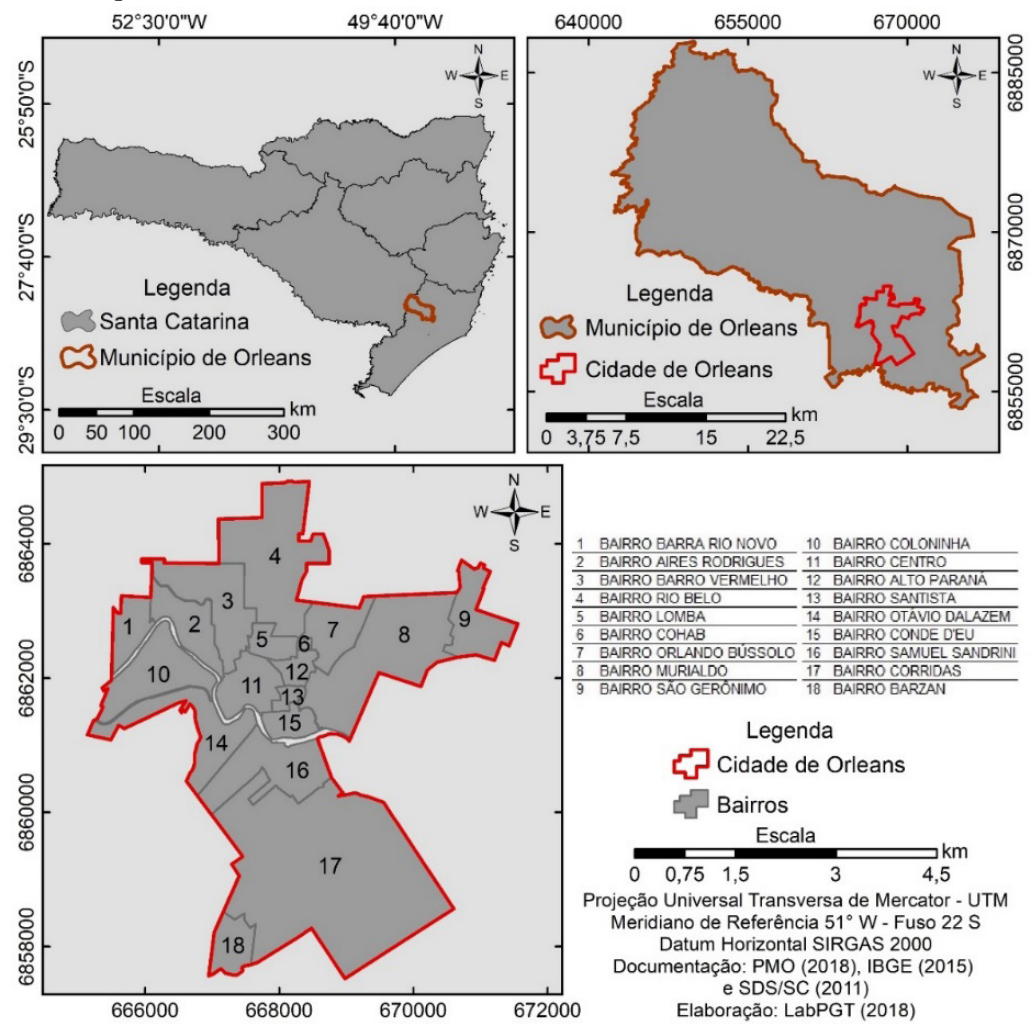

Fonte: LabPGT/UNESC (2018).

O município de Orleans se situa entre o litoral e o planalto catarinense, próximo das encostas da Serra Geral, no Sul de Santa Catarina, com área territorial de $550 \mathrm{~km}^{2}$ e população de 22.587 habitantes (PREFEITURA DE ORLEANS, 2020).
O clima da região é classificado segundo Köppen como subtropical úmido, com verões quentes (ALVARES et al., 2014). O município foi criado por meio da lei estadual $n^{\circ}$. 981, de 30 de agosto de 1913, tendo seu nome definido no 
ano de 1884, pelo Conde d'Eu, em homenagem a sua família francesa e faz parte da Associação dos Municípios da Região Carbonífera - AMREC (WILLEMANN et al., 2020).

Colonizado por descendentes italianos e imigrantes de origem alemã, polonesa, leta e portuguesa (PREFEITURA DE ORLEANS, 2020), Orleans é dotado de rica história, sendo seu território já habitado por índios da etnia Xokleng, sede de uma empresa de colonização, dote de casamento de uma princesa e berço do carvão mineral (WILLEMANN et al., 2020).

O município contempla um valioso acervo históricocultural e integra o roteiro de Imigração do Instituto do Patrimônio Histórico e Artístico Nacional (Iphan), possui atrativos culturais e naturais, com potencial turístico diversificado (WILLEMANN et al., 2020). Além disso, possui a Academia Orleanense de Letras, colégios estaduais, municipais e privados, centro universitário, igrejas e comunidades rurais, vinícola, cachaçaria, associações, entre outros. Entre as atividades econômicas se destaca a agricultura, a pecuária, a indústria e o comércio (PREFEITURA DE ORLEANS, 2020).

A Pastoral da Saúde, cujos membros foram o foco deste estudo, instituída oficialmente no Brasil em 1986, é uma entidade cívico-religiosa e de ação social, sem fins lucrativos, de âmbito nacional e referência internacional. Atua na condição de bem-estar físico, mental e espiritual, enfatizando a saúde integral do ser humano (PASTORAL DA SAÚDE, 2011).

A pesquisa possui natureza quali-quantitativa (AMOROZO; VIERTLER, 2010). Foram realizadas entrevistas semiestruturadas, com auxílio de um formulário adaptado de Rossato et al. (2012), nos meses de fevereiro a julho, no ano de 2019, complementado por turnê guiada (ALBUQUERQUE et al., 2010). Para facilitar a identificação das espécies, foi utilizado registro fotográfico in loco. $\mathrm{O}$ material vegetal coletado foi identificado e armazenado no Herbário Pe. Dr. Raulino Reitz (CRI) da Universidade do Extremo Sul Catarinense (UNESC).

As famílias botânicas foram reunidas pelo sistema Angiosperm Philogeny Group (APG IV, 2016) para as angiospermas e pelo Pteridophyte Philogeny Group (PPG
I, 2016) para as samambaias. A nomenclatura científica das espécies foi confirmada de acordo com o banco de dados The Plant List (2013) (THE PLANT LIST, 2020) e da Lista de Espécies da Flora do Brasil (FLORA DO BRASIL EM CONSTRUÇÃO, 2020).

Para a escolha dos quintais se buscou a coordenadora da Pastoral da Saúde do município que disponibilizou a lista de cadastro, na qual se obteve contato com os agentes e foram entrevistados aqueles que possuíam quintal com cultivo de plantas medicinais e que residiam no perímetro urbano do município de Orleans.

Os dados elencados foram o farmacógeno, a alegação de uso medicinal e as formas de uso, sendo as alegações de uso medicinal organizadas por sistemas orgânicos, utilizando como padrão a nomenclatura dos alvos terapêuticos, utilizada pela Anatomical Therapeutic Chemical (ATC) descrito por Chen et al. (2012). A pesquisa foi aprovada pelo Comitê de Ética com certificado de apresentação para apreciação ética (CAAE) no 2.970.358 (UNESC).

\section{Resultados e Discussão}

Todos os entrevistados $(n=19)$ eram do sexo feminino, agentes da Pastoral da Saúde e a média de idade observada foi de 70 anos. Caballero-Serrano et al. (2019), em seu estudo, constataram que a transmissão do conhecimento está associada a mulheres, especialmente, mães. Sobre a escolaridade, a grande maioria $(63,15 \%)$ não possuía o Ensino Fundamental completo. Rodrigues et al. (2020) também constataram a prevalência do Ensino Fundamental incompleto $(58,92 \%)$ em seu estudo. Quanto ao estado civil, 63,2\% eram viúvas. Quanto à profissão, 31,6\% eram agricultoras e as demais possuíam profissões diversas, como cuidadoras do lar, costureira, professora e cozinheira. Em relação ao estado e município de origem, $78,9 \%$ eram oriundas do estado de Santa Catarina e município de Orleans, as demais $(21,1 \%)$ de localidades vizinhas como Lauro Müller, Grão Pará, Bom Retiro e do Estado do Rio Grande do Sul, municípios de Antônio Prado e Paulo Bento, sendo 89,5\% de descendência italiana. A fisionomia de alguns quintais estudados pode ser visualizada na Figura 2.

Figura 2 - Aspecto fisionômico dos quintais, município de Orleans, Santa Catarina, Sul do Brasil
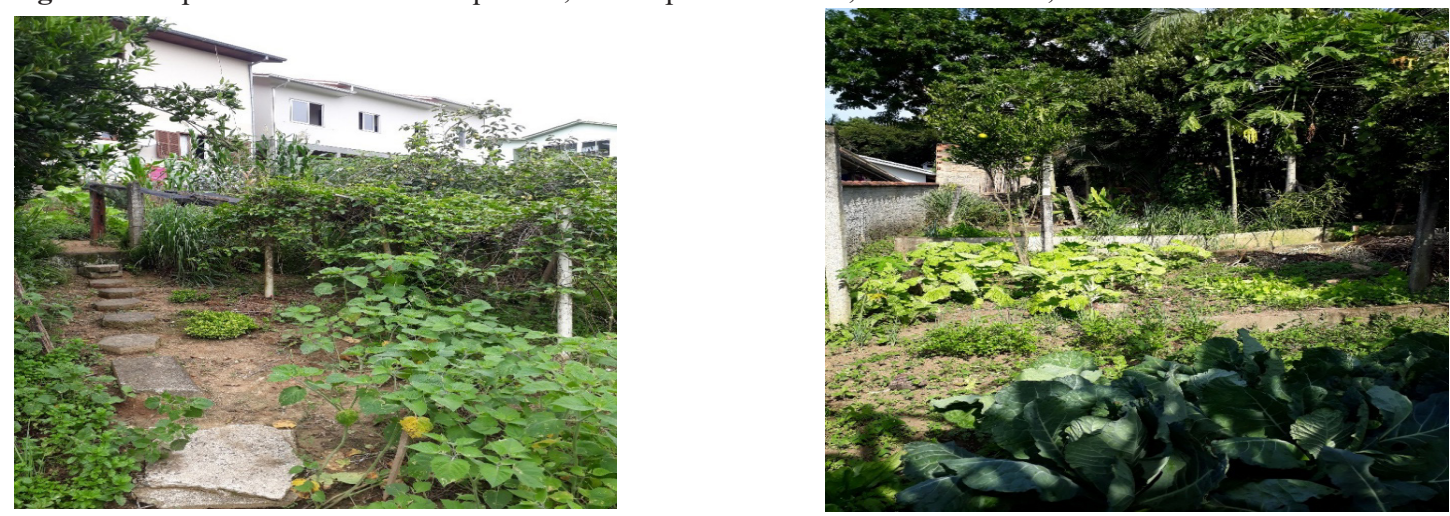

Fonte: Os autores. 
O levantamento etnobotânico registrou 85 espécies, distribuídas em 72 gêneros e 39 famílias botânicas (Quadro 1). Destas, as mais representativas foram Lamiaceae (13 espécies) e Asteraceae (10 espécies), assim como no estudo de Barbosa et al. (2019). Tal predominância pode ser explicada pela ampla distribuição cosmopolita dessas famílias e por incluírem numerosas espécies com compostos bioativos utilizadas para fins terapêuticos (DI STASI et al., 2002; PASA et al., 2019).

Quadro 1 - Relação das espécies medicinais com seus nomes científicos, populares e com suas respectivas famílias botânicas, as quais foram registradas nos quintais urbanos das agentes da Pastoral da Saúde, município de Orleans, Santa Catarina, Sul do Brasil

\begin{tabular}{|c|c|c|}
\hline Família Botânica & Nome Científico & $\begin{array}{c}\text { Nome } \\
\text { Popular }\end{array}$ \\
\hline \multirow{3}{*}{ Amaranthaceae } & $\begin{array}{l}\text { Alternanthera brasiliana (L.) } \\
\text { Kuntze }\end{array}$ & penicilina \\
\hline & Alternanthera tenella Colla & anador \\
\hline & Spinacia oleracea L. & espinafre \\
\hline Amaryllidaceae & Allium sativum L. & alho \\
\hline \multirow[b]{2}{*}{ Apiaceae } & Foeniculum vulgare Mill. & funcho \\
\hline & $\begin{array}{l}\text { Petroselinum crispum (Mill.) } \\
\text { Fuss. } \\
\end{array}$ & salsa \\
\hline Araceae & $\begin{array}{l}\text { Xanthosoma sagittifolium (L.) } \\
\text { Schott. }\end{array}$ & taiá \\
\hline \multirow{10}{*}{ Asteraceae } & Achillea millefolium $\mathrm{L}$. & $\begin{array}{l}\text { mil-em- } \\
\text { ramas }\end{array}$ \\
\hline & Ageratum conyzoides (L.) L. & \begin{tabular}{|c|} 
erva-de-são- \\
joão
\end{tabular} \\
\hline & Bidens pilosa $\mathrm{L}$. & picão-preto \\
\hline & Calendula officinalis L. & calêndula \\
\hline & Chaptalia nutans (L.) Polak. & cachimbinho \\
\hline & Cichorium intybus L. & radiche \\
\hline & $\begin{array}{l}\text { Cynara cardunculus var. } \\
\text { scolymus (L.) Fiori }\end{array}$ & alcachofra \\
\hline & Matricaria chamomilla L. & camomila \\
\hline & Matricaria discoidea DC. & marcela \\
\hline & $\begin{array}{l}\text { Gymnanthenum amygdalinum } \\
\text { (Delile) Sch. Bip. ex Walp. }\end{array}$ & $\begin{array}{l}\text { boldo-do- } \\
\text { chile }\end{array}$ \\
\hline \multirow{2}{*}{ Brassicaceae } & Brassica oleracea L. & couve \\
\hline & Eruca vesicaria (L.) Cav. & rúcula \\
\hline Boraginaceae & Symphytum officinale L. & confrei \\
\hline \multirow[b]{2}{*}{ Cactaceae } & Pereskia aculeata Mill. & ora-pro-nobis \\
\hline & $\begin{array}{l}\text { Pereskia glandiflora Hort. ex } \\
\text { Pfeiff. }\end{array}$ & ora-pro-nobis \\
\hline Caricaceae & Carica papaya L. & mamão \\
\hline Celastraceae & $\begin{array}{l}\text { Monteverdia ilicifolia (Mart. } \\
\text { ex Reissek) Biral }\end{array}$ & $\begin{array}{l}\text { espinheira- } \\
\text { santa }\end{array}$ \\
\hline \multirow{3}{*}{ Commelinaceae } & Commelina erecta $\mathrm{L}$. & trapoeraba \\
\hline & $\begin{array}{l}\text { Dichorisandra thyrsiflora } \\
\text { J.C.Mikan. }\end{array}$ & $\begin{array}{l}\text { cana-do- } \\
\text { brejo }\end{array}$ \\
\hline & Tradescantia zebrina Bosse & trapoeraba \\
\hline Convolvulaceae & Ipomoea batatas (L.) Lam. & batata-doce \\
\hline Costaceae & Costus spiralis (Jacq.) Roscoe. & $\begin{array}{l}\text { cana-do- } \\
\text { brejo }\end{array}$ \\
\hline \multirow{3}{*}{ Crassulaceae } & $\begin{array}{l}\text { Bryophyllum laetivirens } \\
\text { (Desc.) V.V Byalt }\end{array}$ & aranto \\
\hline & $\begin{array}{l}\text { Kalanchoe pinnata (Lam.) } \\
\text { Pers. }\end{array}$ & fortuna \\
\hline & $\begin{array}{l}\text { Sedum dendroideum Moc. \& } \\
\text { Sessé ex DC. }\end{array}$ & bálsamo \\
\hline Cucurbitaceae & Sechium edule (Jacq.) Sw. & chuchu \\
\hline
\end{tabular}

\begin{tabular}{|c|c|c|}
\hline \multirow{4}{*}{ Euphorbiaceae } & $\begin{array}{l}\text { Cnidoscolus aconitifolius } \\
\text { (Mill.) I.M.Johnst. }\end{array}$ & chaya \\
\hline & Euphorbia tirucalli $\mathrm{L}$. & aveloz \\
\hline & Jatropha multifida L. & mercúrio \\
\hline & Manihot esculenta Crantz & aipim \\
\hline Equisetaceae & Equisetum giganteum L. & cavalinha \\
\hline Fabaceae & Cassia angustifolia M. Vahl. & sene \\
\hline \multirow{13}{*}{ Lamiaceae } & Cunila microcephala Benth. & poejo \\
\hline & Melissa officinalis L. & erva-cidreira \\
\hline & Mentha x piperita $\mathrm{L}$. & hortelã \\
\hline & Ocimum basilicum L. & manjericão \\
\hline & Ocimum selloi Benth. & erva-doce \\
\hline & Origanum vulgare L. & orégano \\
\hline & $\begin{array}{l}\text { Plectranthus barbatus } \\
\text { Andrews }\end{array}$ & boldo \\
\hline & Plectranthus ornatus Codd. & $\begin{array}{l}\text { boldo-da- } \\
\text { china }\end{array}$ \\
\hline & Rosmarinus officinalis L. & alecrim \\
\hline & Salvia hispanica L. & chia \\
\hline & Salvia officinalis L. & sálvia \\
\hline & Stachys byzantina K. Koch. & pulmonária \\
\hline & $\begin{array}{l}\text { Tetradenia riparia (Hochst.) } \\
\text { Codd }\end{array}$ & incenso \\
\hline \multirow{2}{*}{ Lauraceae } & Laurus nobilis L. & louro \\
\hline & Persea americana Mill. & abacate \\
\hline \multirow{3}{*}{ Malvaceae } & Não identificada & malva-árvore \\
\hline & $\begin{array}{l}\text { Hibiscus acetosella Welw. ex } \\
\text { Hiern }\end{array}$ & vinagreira \\
\hline & Sida rhombifolia $\mathrm{L}$. & guanxuma \\
\hline Moraceae & Morus nigra L. & amora-preta \\
\hline \multirow{2}{*}{ Myrtaceae } & Eugenia uniflora L. & pitanga \\
\hline & Psidium guajava $\mathrm{L}$. & goiaba \\
\hline Passifloraceae & Passiflora edulis Sims. & maracujá \\
\hline Phyllanthaceae & Phyllanthus tenellus Roxb. & quebra-pedra \\
\hline Piperaceae & $\begin{array}{l}\text { Piper mikanianum (Kunth.) } \\
\text { Steud. }\end{array}$ & pariparoba \\
\hline \multirow{2}{*}{ Plantaginaceae } & Plantago australis Lam. & tansagem \\
\hline & Plantago major L. & tansagem \\
\hline Poaceae & $\begin{array}{l}\text { Cymbopogon citratus (DC.) } \\
\text { Stapf. }\end{array}$ & cana-cidreira \\
\hline Pteridaceae & Adiantum raddianum C. Presl & avenca \\
\hline Ranunculaceae & Ranunculus sceleratus L. & erva-ciática \\
\hline \multirow[t]{2}{*}{ Rosaceae } & $\begin{array}{l}\text { Eriobotrya japonica (Thunb.) } \\
\text { Lindl. }\end{array}$ & $\begin{array}{l}\text { ameixa- } \\
\text { amarela }\end{array}$ \\
\hline & Rosa sp. & \begin{tabular}{|l|} 
rosa-branca \\
\end{tabular} \\
\hline \multirow{3}{*}{ Rutaceae } & $\begin{array}{l}\text { Citrus aurantiifolia (Christm.) } \\
\text { Swingle. }\end{array}$ & laranja-lima \\
\hline & Citrus limon (L.) Osbeck & limão \\
\hline & Ruta graveolens L. & arruda \\
\hline Smilacaceae & Smilax aspera $\mathrm{L}$. & salsa-parrilha \\
\hline Solanaceae & Solanum aethiopicum L. & jiló \\
\hline \multirow{3}{*}{ Verbenaceae } & Aloysia citriodora Palau & hierba-luisa \\
\hline & $\begin{array}{l}\text { Aloysia gratissima (Gillies \& } \\
\text { Hook.) Tronc. }\end{array}$ & erva-santa \\
\hline & $\begin{array}{l}\begin{array}{l}\text { Stachytarpheta cayennensis } \\
\text { (Rich.) Vahl }\end{array} \\
\end{array}$ & gervão \\
\hline Vitaceae & $\begin{array}{l}\text { Cissus verticillata }(\mathrm{L} .) \\
\text { Nicolson \& C.E.Jarvis }\end{array}$ & insulina \\
\hline Violaceae & Viola odorata L. & $\begin{array}{l}\text { violeta-de- } \\
\text { jardim }\end{array}$ \\
\hline \multirow{2}{*}{ Xanthorrhoeaceae } & Aloe arborescens Mill. & babosa \\
\hline & Aloe vera (L.) Burm.f. & babosa \\
\hline \multirow{3}{*}{ Zingiberaceae } & $\begin{array}{l}\text { Alpinia zerumbet (Pers.) B.L. } \\
\text { Burtt \& R.M. Sm. }\end{array}$ & noz-moscada \\
\hline & Curcuma longa $\mathrm{L}$. & cúrcuma \\
\hline & Zingiber officinale Roscoe & gengibre \\
\hline
\end{tabular}

Fonte: Dados da pesquisa. 
Do total, 55 (64,7\%) das espécies levantadas são exóticas, denotando ainda pouco conhecimento de espécies nativas do Brasil (35,3\%) com potencial medicinal. Em relação ao farmacógeno, a folha $(84,7 \%)$ se sobressaiu como o órgão da planta mais utilizado nas preparações terapêuticas (Figura 3 ), sendo também coincidente no estudo realizado por Neto e Gomes (2018), que relatam a folha como a parte da planta mais utilizada nas preparações medicinais.

Figura 3 - Partes das plantas (farmacógenos) relacionadas ao uso medicinal, informadas pelas entrevistadas da Pastoral da Saúde, município de Orleans, Santa Catarina, Sul do Brasil

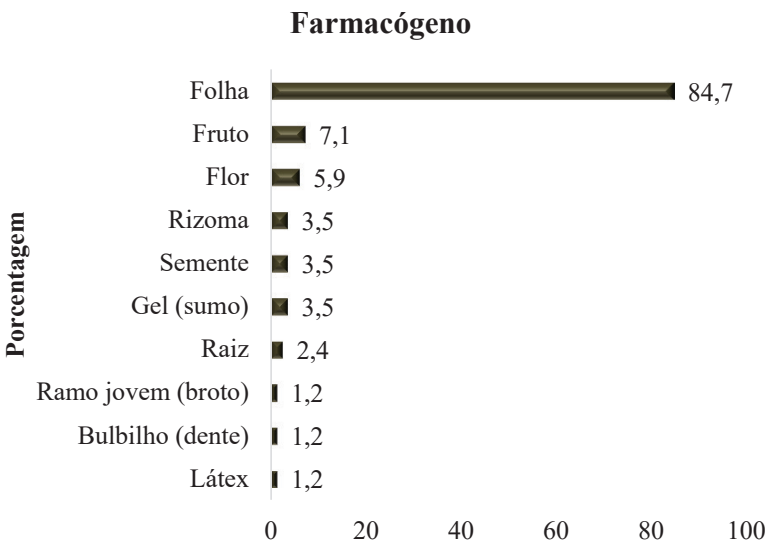

Fonte: Dados da pesquisa.

O estado do farmacógeno (fresco ou seco) e a forma de uso (interno ou externo) mais utilizados foram o farmacógeno fresco $(96,5 \%)$ e o uso interno $(88,2 \%)$, respectivamente, prática observada igualmente por Giraldi e Hanazaki (2010), em que $60 \%$ das plantas são utilizadas frescas e $82 \%$ de uso interno, quando realizaram estudo etnobotânico sobre o uso e o conhecimento tradicional de plantas medicinais na comunidade açoriana do Sertão do Ribeirão, no município de Florianópolis, Santa Catarina. As formas farmacêuticas mais citadas foram o infuso $(50,6 \%)$ e o decocto $(49,4 \%)$ (Figura 4). Outros autores encontraram resultados similares, como no estudo de Tatagiba et al. (2019), em que as preparações medicinais por chás (decocção e infusão) foram os métodos de preparo mais utilizados.

Figura 4 - Formas de uso e preparo realizadas pelas informantes da Pastoral da Saúde, município de Orleans, Santa Catarina, Sul do Brasil

\section{Farmacógeno}

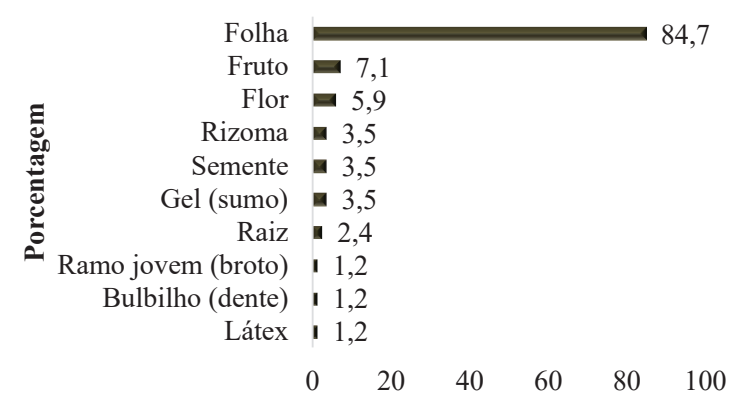

Fonte: Dados da pesquisa.
As formas de preparo denominadas pelas agentes como "socado" e "queimada" significam, respectivamente: triturar o farmacógeno com algum objeto e aquecer no fogo junto com canela/açúcar mascavo. Já o "suco liquefeito" corresponde à trituração realizada no liquidificador, diferenciando do suco comum (Figura 4).

Vale ressaltar que cada entrevistada possuía o seu próprio método de preparação, cuja nomenclatura e forma de preparo nem sempre eram padronizadas, podendo variar em função da quantidade e qualidade do recurso botânico utilizado, e de acordo com a intensidade da enfermidade a ser tratada. Essa autonomia é identificada por Morais et al. (2018), que consideraram o cultivo de plantas medicinais nos quintais como uma alternativa à redução de custos com medicamentos industrializados, nos casos de combate imediato de algum malefício de menor risco, além de ser um tratamento natural, evitando possíveis efeitos colaterais.

Das 85 espécies citadas, resultaram 170 usos medicinais, os quais foram divididos em 10 sistemas orgânicos, prevalecendo, neste estudo, aqueles para o trato alimentar e metabolismo (25,88\%), as relacionadas ao sangue e aos órgãos hematopoiéticos (12,94\%) e para o tratamento do sistema respiratório (11,76\%) (Quadro 2). Cerqueira et al. (2020) encontraram resultados aproximados para doenças gastrointestinais $(31,1 \%)$, respiratórias $(7,1 \%)$ e cardiovasculares $(3,0 \%)$.

Quadro 2 - Frequência de usos medicinais por sistemas orgânicos das espécies registradas nos quintais urbanos das agentes da Pastoral da Saúde, município de Orleans, Santa Catarina, Sul do Brasil

\begin{tabular}{|l|c|c|}
\hline \multicolumn{1}{|c|}{ Sistemas Orgânicos* } & N & \% \\
\hline Trato Alimentar e Metabolismo & 44 & $25,88 \%$ \\
\hline Sangue e Órgãos Hematopoiéticos & 22 & $12,94 \%$ \\
\hline Sistema Respiratório & 20 & $11,76 \%$ \\
\hline Sistema Nervoso Central & 19 & $11,18 \%$ \\
\hline Sistema Geniturinário e Hormônios Sexuais & 16 & $9,41 \%$ \\
\hline Anti-infecciosos Gerais para Uso Sistêmico & 14 & $8,24 \%$ \\
\hline Dermatológicos & 12 & $7,06 \%$ \\
\hline Sistema Musculoesquelético & 12 & $7,06 \%$ \\
\hline Sistema Cardiovascular & 8 & $4,71 \%$ \\
\hline Agentes Antineoplásicos e Imunomoduladores & 3 & $1,76 \%$ \\
\hline
\end{tabular}

*Variável formada por 170 usos medicinais.

Fonte: Dados da pesquisa.

Com relação às espécies com maior número de indicações terapêuticas estão alecrim (07), babosas (06), mil-emramas, rosa-branca e limão (05). Conforme relatado pelas entrevistadas, o alecrim (Rosmarinus officinalis) está relacionado com enfermidades do sistema cardio/circulatório, para evitar coágulos no sangue, pressão arterial - hipertensão, sistema nervoso, como dor de cabeça, e sistema respiratório, como resfriados. Em seu estudo, Duarte et al. (2020) também verificaram que a maioria das espécies possui mais de uma indicação terapêutica, como no caso de $R$. officinalis, que foi indicado para artrite, depressão e coração. Oliveira e Veiga 
(2019) relataram que $R$. officinalis possui impacto positivo na saúde humana, uma vez que apresenta propriedades terapêuticas importantes como antioxidantes, antimicrobiana e anti-inflamatória, que se configuram como excelente opção de tratamento e prevenção de problemas que comprometem a saúde. As babosas (Aloe vera e A. arborescens) são utilizadas, em sua maioria, topicamente para tratamento de feridas, queimaduras e, ainda, uso interno para males do estômago (gastrite), na forma de suco liquefeito. Conforme o estudo de Cagni e Lubi (2018), A. vera apresenta mucilagem no parênquima de suas folhas com propriedades cicatrizantes que estimulam o crescimento dos tecidos e a regeneração celular, o que contribui para a reparação tecidual, auxiliando, portanto, na cicatrização epitelial. Nesta direção, Sousa et al. (2020) apontaram atividades biológicas da babosa com ação antinociceptiva, anti-inflamatória, cicatrizante e imunomoduladora. A mil-em-ramas/pronto-alívio (Achillea millefolium) é utilizada para o alívio de dores de cabeça e muscular, como calmante e, também, para o sistema digestório (fígado e estômago). De acordo com Andrade (2018), A. millefolium atua como neuroprotetora, antioxidante, antiinflamatória, melhorando as funções motoras e cognitivas, além de proporcionar benefícios quanto à progressão da doença de Parkinson. A rosa-branca (Rosa sp.) é citada popularmente para uso tópico em casos de inflamação/alergia nos olhos e seu uso é ratificado por Souza e Dória (2016), em seu levantamento etnobotânico, para a mesma afecção. O limão (Citrus limon) é conhecido por suas diversas finalidades, em especial, para "limpar/afinar o sangue" (depurativo). Everton et al. (2018) apontam que as partes comumente utilizadas do limão (folhas e frutos) demonstraram atividade antimicrobiana e antioxidante, bem como elevado teor de fenóis totais, o que reforça as informações advindas da medicina popular.

Quanto ao número de citações, a hortelã (09) foi a espécie mais citada, seguida de alecrim e quebra-pedra (08) e de orapro-nobis e gengibre (07). A hortelã (Mentha $x$ piperita) foi citada para alívio de males no estômago e como calmante. Avaliando o efeito antimicrobiano Rempel et al. (2019) constaram que a hortelã está entre as espécies com maior número de publicações comprovando sua eficácia medicinal. Já Oliveira et al. (2020) confirmam a atuação da hortelã no sistema digestório. A quebra-pedra (Phyllanthus tenellus) foi indicada para cálculo renal ou pedra na vesícula, sendo estes agravos semelhantes aos relatados por Ferreira et al. (2016), que apontam a planta para infecção urinária, rim e cálculo renal. A ora-pro-nobis (Pereskia aculeata e P. grandiflora) são utilizadas como alimento - in natura, como fonte de proteína, no tratamento/prevenção de anemia e para imunidade. Faria et al. (2019) confirmam esta indicação popular, por meio da análise química de suas folhas, relatando que $P$. aculeata serve como excelente fonte proteica, podendo ser incluída na alimentação da população, porém adverte que a quantidade de proteína varia de acordo com a região. O gengibre (Zingiber officinale), por suas propriedades anti-inflamatórias, foi indicado para o tratamento de enfermidades do trato respiratório (garganta, tosse, gripe, asma). Em relação aos efeitos terapêuticos do gengibre, Sousa et al. (2019), atestam que esse possui atividades farmacológicas diversas como antieméticas, antitumorais, antioxidantes, anti-inflamatórias e antimicrobianas.

Referente ao tempo de uso das plantas medicinais mencionado pelas entrevistadas, $73(85,9 \%)$ são conhecidas há mais de 50 anos, o que sinaliza potenciais espécies para futuros levantamentos mais detalhados, atendendo aos critérios da Agência Nacional de Vigilância Sanitária (ANVISA) quanto ao registro com base na tradicionalidade de uso superior a 30 anos, para produtos tradicionais fitoterápicos (ANVISA, 2014). A herança cultural, de acordo com Rodrigues et al. (2020), é a grande responsável pelo acúmulo de conhecimentos obtido por meio das pessoas mais idosas.

\section{Conclusão}

Este estudo reflete o contexto cultural e ambiental enraizado, revelando a importância das investigações etnobotânicas e dos quintais como forma social, que pode contribuir para a manutenção dos espaços urbanos, por meio do registro do conhecimento popular.

Embora com predomínio de espécies exóticas, algumas espécies nativas já estão sendo domesticadas, o que poderá incluir plantas em cultivo de pequena escala em futuro próximo.

Os recursos vegetais utilizados com fins medicinais se apresentam úteis também à conservação in situ da agrobiodiversidade, uma vez que nos espaços representados pelos quintais estão presentes uma inestimável riqueza de espécies.

\section{Agradecimentos}

Ao Programa de Suporte à Pós-Graduação de Instituições de Ensino Comunitárias de Educação Superior (PROSUC/ CAPES) pela bolsa de Mestrado concedida à primeira autora.

\section{Referências}

ALBUQUERQUE; U.P. et al. Métodos e técnicas para coleta de dados etnobiológicos. In: ALBUQUERQUE, U.P.; LUCENA, R.F.P.; CUNHA, L.V.F.C. Métodos e técnicas na pesquisa etnobiológica e etnoecológica. RecifeNUPEEA, 2010. p.39-64.

ALVARES, C.A. et al. Köppen's climate classification map for Brazil. Meteorologische Zeitschrift, v.22, n.6, p.711-728, 2014. doi: 10.1127/0941-2948/2013/0507.

AMOROZO, M.C.M.; VIERTLER, R. B. A abordagem qualitativa na coleta e análise de dados em etnobiologia e etnoecologia. In: ALBUQUERQUE, U. P.; LUCENA, R. F. P.; CUNHA, L. V. F. C. Métodos e técnicas na pesquisa etnobiológica e etnoecológica. Recife: NUPEEA, 2010. p.67-82.

ANDRADE, I. G. S. Beneficios e riscos das plantas medicinais na doença de Parkinson. 2018. 62 f. Dissertação (Mestrado integrado em Ciências Farmacêuticas). Universidade de Coimbra, 2018.

APG IV. An update of the Angiosperm Phylogeny Group 
classification for the orders and families of flowering plants: APG IV. Botanical Journal of the Linnean Society, v.181, p.1-20, 2016. doi: 10.1111/boj.12385.

ÁVILA, J. V. C. et al. Agrobiodiversity and in situ conservation in quilombola home gardens with different intensities of urbanization. Acta Botanica Brasilica, Belo Horizonte, v.31, n.1, p.1-10, 2017. doi: 10.1590/0102-33062016abb0299.

BARBOSA, C. S. et al. Plantas medicinais cultivadas em quintais no bairro de São Raimundo, da cidade de Manaus, AM. Revista Terceira Margem Amazônia, v.4, n.12, p.122-141, 2019.

BENTO, L. V. O. et al. Agrobiodiversidade dos quintais urbanos de Rio Branco, AC. VII CONNEPI - Congresso Norte-Nordeste de Pesquisa e Inovação. 2012.

CABALLERO-SERRANO, V. et al. Traditional ecological knowledge and medicinal plant diversity in Ecuadorian Amazon home gardens. Global Ecology and Conservation, v. 17, p. e00524, 2019. doi: 10.1016/j.gecco.2019.e00524.

CAGNI, T. F.; LUBI, N. Aloe vera no reparo tecidual. Revista Eletrônica Biociências, Biotecnologia e Saúde, v. 11, n. 20, p. 33-42, 2018.

CERQUEIRA, T. M. G. et al. The use of medicinal plants in Maceió, Northeastern Brazil: an ethnobotanical survey, Medicines, v.7, n.2, p.7, 2020. doi: 10.3390/medicines 7020007

CHEN, L. et al. Predicting anatomical therapeutic chemical (ATC) classification of drugs by integrating chemical-chemical interactions and similarities. PLoS One, v. 7, n. 4, p. e35254, 2012.

DIOCESE DE TUBARÃO. Paróquias. Paróquia Santa Otília Orleans. 2019.

DI STASI, L. C. et al. Lamiales medicinais. In: DI STASI, C. et al. Plantas medicinais na Amazônia e na Mata Atlântica. 2.ed. São Paulo: UNESP, 2002. 406 p.

DI STASI, L. C. et al. Asterales medicinais. In: DI STASI, L. C. et al. Plantas medicinais na Amazônia e na Mata Atlântica. 2.ed. São Paulo: UNESP, 2002. 463 p.

DUARTE, A. M. et al. Saberes e Práticas populares no uso de plantas medicinais em espaço urbano no planalto Sul Catarinense. Revista Brasileira de Agroecologia, v.15, n.1, p. 28, 2020. doi: 10.33240/rba.v15i1.22978.

EVERTON, G. O. et al. Atividade antioxidante e antimicrobiana das folhas e frutos de Citrus limon (L.) Burn (limão siciliano). Revista Cubana de Plantas Medicinales, v.23, n.4, 2018.

FARIA, S.; CATUNDA, K.; OLIVEIRA, L. M. Análise química de folhas de ora-pro-nobis (Pereskia aculeata). Saber Cientifico, v.8, n.2, p.99-103, 2019. doi: 10.22614/resc-v8-n2-1255.

FERREIRA, L. B.; RODRIGUES, M. O.; COSTA, J. M. Etnobotânica das plantas medicinais cultivadas nos quintais do bairro de Algodoal em Abaetetuba/PA.

Revista Fitos, v.10, n.3, p.220-372, 2016. doi: 10.5935/24464775.20160020.

FLORADO BRASIL 2020 EM CONSTRUÇÃO. Jardim Botânico do Rio de Janeiro. SiBBr. Rio de Janeiro, 2010. Disponível em: http://floradobrasil.jbrj.gov.br/. Acesso em: 22 ago. 2020.

GIRALDI, M.; HANAZAKI, N. Uso e conhecimento tradicional de plantas medicinais no Sertão do Ribeirão, Florianópolis, SC, Brasil. Acta Botanica Brasilica, v.24, n.2, p.395-406, 2010. doi: 10.1590/S0102-33062010000200010.

MORAIS, G. F. et al. Agrobiodiversidade manejada em quintais agroflorestais urbanos: cultivo de plantas medicinais. Cadernos de Agroecologia, v.13, n.2, p. 1-17, 2018.

NETO, L. A. G.; GOMES, F. T. L. Levantamento etnobotânico de plantas medicinais utilizadas pela população do município de Oliveira Fortes - MG. Perspectivas Online: Biológicas \& Saúde. v.8, n.27, p.1-17, 2018.

OLIVEIRA, D. F. et al. Fitoterápicos que atuam no sistema digestório: possíveis mecanismos de ação. Brazilian Journal of health review, v.3, n.3, p.4274-4297, 2020. doi: 10.34119/ bjhrv3n3-030.

OLIVEIRA, J. C. A.; VEIGA, R. S. Impacto do uso do alecrim (Rosmarinus officinalis L.) para a saúde humana. Brazilian Journal of Natural Sciences, v.1, n.2, p. 12, 2019. doi: 10.31415/ bjns.v2i1.40.

PASA, M. C. et al. Medicinal plants in cultures of afro-descendant communities in Brazil, Europe and Africa. Acta Botanica Brasilica, v. 33, n.2, p.340-349, 2019.

PASTORAL DA SAÚDE. Manual do Agente da Pastoral da Saúde. Subsídios Pastoral da Saúde: Diretrizes da Pastoral da Saúde, n.1, 2.ed. 2011.

PERNA, T. A.; LAMANO-FERREIRA, A. P. N. 2014. Revisão bibliométrica sobre o cultivo de plantas medicinais em quintais urbanos em diferentes regiões do Brasil (2009-2012). UNOPAR Científica Ciências Biológicas e da Saúde, v.16, n.1, p.61-70, 2014. doi: 10.17921/2447-8938.2014v16n1p\%25p.

PPG I - The Pteridophyte Phylogeny Group. A communityderived classification for extant lycophytes and ferns. Journal of Systematics and Evolution, v.54, n.6, p.563-603, 2016.

PRADEICZUK, A. et al. Urban ethnobotany: a case study in neighborhoods of different ages in Chapecó, Santa Catarina State. Acta Botanica Brasilica, v.31, n.2, p.276-285, 2017. doi: 10.1590/0102-33062017abb0080.

PREFEITURA DE ORLEANS. Características. Santa Catarina, Brasil. Disponível em: http://www.pmo.sc.gov.br/index.php/ inicio/caracteristicas. Acesso em: 27 ago. 2020.

REMPEL, C. et al. Efeito antimicrobiano de plantas medicinais: uma revisão de estudos científicos. Revista Ibero-Americana de Ciências Ambientais, v.10, n.4, p.57-82, 2019. doi: 10.6008/ CBPC2179-6858.2019.004.0006.

RITTER, M. R. et al. Bibliometric analysis of ethnobotanical research in Brazil (1988-2013). Acta Botanica Brasilica, v.29, n.1, p.113-119, 2015. doi: 10.1590/0102-33062014abb3524.

RODRIGUES, T. A. et al. A valorização das plantas medicinais como alternativa à saúde: um estudo etnobotânico. Revista Ibero Americana de Ciências Ambientais, v.11, n.1, p.411- 428, 2020. doi: 10.6008/CBPC2179-6858.2020.001.0037.

ROSSATO, A. E. et al. Fitoterapia racional: aspectos taxonômicos, agroecológicos, etnobotânicos e terapêuticos. Florianópolis: DIOESC, v.1. 2012. 211p.

ROSSATO, A. L. Uso de Plantas Medicinais: Cultura Popular na experiência da Pastoral da Saúde da Paróquia São Paulo Apóstolo em Criciúma, SC. 2018. 60 f. Monografia (Trabalho de Conclusão de Curso de História) - Universidade do Extremo Sul Catarinense - SC, 2018.

SIVIERO, A. et al. Plantas medicinais em quintais urbanos de Rio Branco, Acre. Revista Brasileira de Plantas Medicinais, v.14, n.4, p.598-610, 2012. doi: 10.1590/S1516-05722012000400005.

SOUSA, E. A. O.; NEVES, E. A.; ALVES, C. R. Potencial terapêutico de Aloe vera (Aloe barbadensis): uma breve revisão. Revista Virtual de Química, v.12, n.2, p.378-388, 2020.

SOUSA, J. P. Breve relato sobre os efeitos terapêuticos do 
gengibre (Zingiber officinale Roscoe). Revista da Faculdade de Educação e Meio Ambiente, v.10, n.1, p.44-53, 2019. doi: 10.31072/rcf.v10iedesp.785.

SOUZA, L.; DÓRIA, K. M.A. B. V. S. Levantamento etnobotânico de plantas medicinais no bairro Massaguaçu, Caraguatatuba - SP. Unisanta BioScience, v.5, n.4, p. 335-345, 2016. doi: 10.18066/ revistaunivap.v22i40.901.

TATAGIBA, S. D.; SOUSA, I. S.; OLIVEIRA, A. E. W. Etnobotânica de plantas medicinais na Região de Integração do
Rio Tapajós, Comunidade do Bairro Maria Magdalena, Município de Itaituba, Pará, Brasil. Biota Amazônia, v.9, n.4, p.41-49, 2019. doi: 10.18561/2179-5746/biotaamazonia.v9n4p41-49.

THE PLANT LIST. Version 1.1: A working list of all plant species, Sep. 2013. Available at: http://www.theplantlist.org. Accessed in: 17th Aug. 2020.

WILlEMANN, E. et al. Plano Municipal de Turismo de Orleans 2020-2030. Orleans/SC: Editora: UNIBAVE, 2020. 104 p. 